\title{
Prenatal and early postnatal measures of brain development and childhood sleep patterns
}

\author{
Desana Kocevska ${ }^{1}$, Maria E. Verhoeff ${ }^{1}$, Selma Meinderts ${ }^{2}$, Vincent W.V. Jaddoe ${ }^{3}$, Frank C. Verhulst ${ }^{1}$, Sabine J. Roza ${ }^{4}$, \\ Maartje P. Luijk ${ }^{5}$ and Henning Tiemeier ${ }^{3}$
}

BACKGROUND: Brain development underlies maturation of sleep patterns throughout childhood. Intrauterine head growth-marker of early neurodevelopment-has not been associated with childhood sleep characteristics. We explored associations between ultrasonographic measures of prenatal and early postnatal neurodevelopment and childhood sleep. METHODS: A total of 6,808 children from a population-based birth cohort (Generation R) were included. Head circumference $(\mathrm{HC})$ and lateral ventricles size were assessed with midand late-pregnancy fetal ultrasounds, and with cranial ultrasound 3-20 weeks postnatally. Mothers reported children's sleep duration at 2 and 3 years, and sleep problems at 1.5, 3, and 6 years.

RESULTS: Larger ventricular size, but not $\mathrm{HC}$, was related to longer sleep duration at 3 years $(\beta=0.06 \mathrm{~h}, 95 \%$ confidence interval (Cl): 0.02; 0.10 in late-pregnancy and $\beta=0.11 \mathrm{~h}, 95 \% \mathrm{Cl}$ : $0.02 ; 0.20$ in early infancy, mid-pregnancy parameters were unrelated to sleep duration). Larger HC in mid-pregnancy was associated with a reduced risk for being a "problematic sleeper" up to the age of 6 years (odds ratio (OR): $0.94,95 \% \mathrm{Cl}$ : 0.89; 0.99). Consistently, children with larger $\mathrm{HC}$ in early infancy were less likely to be "problematic sleepers" at 3 and 6 years.

CONCLUSIONS: This study shows that variations in fetal and neonatal brain size may underlie behavioral expression of sleep in childhood. Albeit small effect estimates, these associations provide evidence for neurodevelopmental origins of sleep.

$\mathbf{N}$ ewborns of different species undergo developmental maturation of sleep patterns (1). The maturation of sleep patterns is considered to be an important developmental milestone for the human infant, e.g., decrease in total sleep duration and napping (2). As brain development is intrinsically related to the sleep-wake process, it may underlie variations in sleep patterns observed throughout childhood.
A full understanding of how markers of early neurodevelopment are related to childhood sleep patterns is lacking.

The second and third trimester of intrauterine life, as well as early infancy form a vulnerable period for the development of the brain (3). Rapid maturational changes of sleep expression go alongside neurodevelopment of the fetus (4) and the infant (5), thus impaired brain development during this period could later affect sleep patterns and problems. Several prenatal exposure studies lend support for this hypothesis. Prenatal tobacco (6) and alcohol (7) exposure is related to disturbed neonatal sleep, and maternal mood disturbances during pregnancy are related to disturbed sleep patterns in toddlerhood (8). Birth outcomes have also been tested as determinants of childhood sleep patterns (6). Children born preterm or small for gestational age have disturbed sleep patterns in infancy (9) and childhood (6). However, these birth parameters are relatively crude measures of intrauterine growth and provide little or no information on fetal brain development. Intrauterine head growth is a reliable indicator of early neurodevelopment reflecting both genetic and environmental effects, but it has not been related to childhood sleep.

Previous studies on the current sample $(10,11)$ and other samples $(12,13)$ show that measures of early brain development assessed with the fetal and neonatal cranial ultrasound are predictive of later neurodevelopmental outcomes. Prenatal head circumference is a reliable proxy for the brain volume growth of the fetus (14) associated with later cognitive functioning (15). The size of the ventricular system is another marker of early brain development $(10,13,14)$, providing information on the growth of the cerebral hemispheres both prenatally and in neonates.

This study explores the associations of prenatal and neonatal ultrasonographic measures of brain growth with childhood sleep patterns in a large sample from the general population. We hypothesized that a more advanced brain maturation, indicated by larger head circumference and larger lateral ventricles within the normal range, is associated with longer sleep duration and less sleep problems up to 6 years of

\footnotetext{
${ }^{1}$ Department of Child and Adolescent Psychiatry, Erasmus Medical Center, Rotterdam, The Netherlands; ${ }^{2}$ Generation R Study Group; ${ }^{3}$ Department of Epidemiology, Erasmus Medical Center, Rotterdam, The Netherlands; ${ }^{4}$ Department of Psychiatry, Erasmus Medical Center, Rotterdam, The Netherlands; ${ }^{5}$ Department of Psychology, Education and Child Studies, Erasmus University Rotterdam, Rotterdam, The Netherlands. Correspondence: Henning Tiemeier (h.tiemeier@erasmusmc.nl)

Received 12 September 2017; accepted 22 November 2017; advance online publication 17 January 2018. doi:10.1038/pr.2017.318
} 


\section{Early head growth and sleep in childhood Articles}

age. As adversities in early neurodevelopment are associated with childhood behavioral problems (16), which in turn are often comorbid with sleep problems (17), we also tested whether the associations are independent of behavioral problems.

\section{METHODS}

\section{Study Population}

We conducted our study within the ongoing Generation R Study, which follows children born between April 2002 and January 2006 in Rotterdam, the Netherlands. The study has previously been described in detail (18). The Medical Ethics Committee of Erasmus Medical Centre approved the study, and written informed consent was obtained from all parents.

Prenatal ultrasounds were performed in 8.209 women in midpregnancy and in 8.270 women in late pregnancy. Neonatal head circumference up to 2 months of age was assessed in 5,558 children. Additional detailed ultrasound measurements were obtained in a random subsample of Dutch children $(n=776)$. (11) Sleep patterns were assessed in 6,808 ( $n=723$ with postnatal cranial ultrasound) of these children $(72 \%$ follow-up, more details in Supplementary Figure S1 online) after excluding twins $(n=132)$ and those children that were lost to follow-up $(n=425)$. We used all available information, and thus the sample size differs per analyses. The children assessed at different time points did not differ with respect to brain size, sleep indices or other sociodemographic characteristics (Supplementary Table S1).

\section{Determinants}

Fetal ultrasound. Fetal ultrasound measurements were carried out in early, mid- and late pregnancy using the Aloka model SSD-1700 (Tokyo, Japan) or the ATL-Philips Model HDI 5000 (Seattle, WA), with standardized ultrasound procedures. Gestational age was established using data from the first fetal ultrasound examination (19). As the size of the ventricles can be reliably measured only from the beginning of the second trimester, only mid-pregnancy (average gestational age $21(18-24)$ weeks) and late pregnancy (average gestational age $30(25-39)$ weeks) measures were used. The atrial width of the lateral ventricles was measured as the widest diameter of the atrium of one of the lateral ventricles in an axial plane (10). Based on the reference growth curves from the whole study population, gestational age-adjusted standard deviation (SD) head circumference scores were constructed, which represent the equivalent of $z$-scores. (19) The intra- and interobserver reliability of fetal biometry measurements in early pregnancy were excellent (intraclass correlation coefficient $>0.99$ ).

Postnatal head circumference. Infant head circumference was measured at Community Health Centers (4.5 \pm 0.9 postnatal weeks) using standard procedures. Values were expressed as ageand gender-adjusted SD scores using Dutch reference growth curves (20). During the research center visit for the infant cranial ultrasound $(6.9 \pm 1.9$ postnatal weeks) we measured the fronto-occipital head circumference $(\mathrm{cm})$ at its maximum diameter through the glabella and occiput to the nearest $0.1 \mathrm{~cm}$, using a flexible measuring tape (10).

Infant cranial ultrasound. Postnatal cranial ultrasounds were performed at $6.9 \pm 1.9$ postnatal weeks using a commercially available multifrequency electronic transducer $(3.7-9.3 \mathrm{MHz})$ with a scan angle of $146^{\circ}$ (Voluson 730 Expert; GE Healthcare, Waukesha, WI). The infants were placed in a supine position, using a probe on the anterior fontanel and a volume box at the level of foramen Monro in a symmetrical coronal section. The obtained data were analyzed with MNI Display software (Montreal Neurological Institute, McGill University, Quebec, Canada). Four raters manually traced left and right lateral ventricles using a mouse-driven cursor, after intensive training with an experienced ultrasonographer, as previously described in detail (10). Intraobserver intraclass correlation coefficients were all above 0.99 , and interobserver intraclass correlation coefficients were above 0.95 .

\section{Outcomes}

Sleep duration. The usual bedtimes, wake times, and the amount of daytime sleep (categories ranging between $<30 \mathrm{~min}$ and $>2.5 \mathrm{~h}$ ) were reported by the parents when children were 2 and 3 years old. Sleep duration was calculated as hours of sleep per $24 \mathrm{~h}$ by adding nighttime and daytime sleep. At 3 years of age, separate reports for weekdays and weekends were available, and a weighted average sleep duration was computed $((5 \times$ weekday $+2 \times$ weekend $) / 7)$.

Sleep problems. At 1.5, 3, and 6 years, the parents answered five items measuring dyssomnia symptoms (Doesn't want to sleep alone; Has trouble getting to sleep; Resists going to bed at night; Sleeps less than most kids during day and/or night; Wakes up often at night) from the Child Behavior Checklist (1.5-5) on a three-point Likert scale $(0-$ not true, 1 -somewhat or sometimes true, or 2 -very or often true). (21) In line with a previous study, (22) we did not include the Child Behavior Checklist parasomnia items in the scores, as parasomnias might have a different neurodevelopmental origins. Sleep problems sum scores (range $0-10$ ) were computed by summing the dyssomnia items. As the sleep problems scores at each time point were strongly right skewed, we categorized children in the highest quartiles as "problematic sleepers" and studied the remainder as the reference group. Because of the developmental changes in sleep patterns up to 6 years, the cutoff point of the highest quartile of sleep problems varies with age (e.g., $>2.5$ points at age 1.5 years, and $>2$ points at 3 and 6 years).

\section{Covariates}

Child characteristics. Estimated fetal weight was calculated using the formula by Hadlock et al (23). Information on sex, date of birth, gestational age, birth weight, and Apgar score 5 min after birth was obtained from midwives and hospital registries. Postnatal ( $4.7 \pm 0.9$ weeks) height and weight was measured at the Community Health Centers and was expressed in age- and gender-adjusted SD scores using Dutch reference growth curves (20). Child's ethnicity was based on parent's country of birth and categorized into: Dutch (Netherlands), other Western (other European countries, United States, Canada, Australia, and Japan), Mediterranean (Turkey and Morocco), Caribbean (Dutch Antilles and Surinam), or other non-Western (Africa, Asia, non-Western America, and Cape Verde). (24) Children internalizing and externalizing problems at age 3 years were assessed with the Child Behavior Checklist (21).

Maternal characteristics. Maternal age, education, and parity was assessed with questionnaires at enrollment. Educational level was classified into high, intermediate, or low (24). History of tobacco smoking was obtained by questionnaires in early, mid-, and late pregnancy and was categorized into: "never smoked", "stopped smoking when pregnancy was known," and "continued smoking during pregnancy." Maternal psychiatric symptoms during pregnancy were assessed using the Global Severity Index from the Dutch version of the Brief Symptom Inventory (25).

\section{Statistical Analyses}

We tested the associations of early brain growth, i.e., fetal and infant brain ultrasound measures of head and ventricular size with sleep duration using linear regression. To study the association between early brain growth and sleep problems up to 6 years of age, we used logistic and liner regression models for each time point separately, and general estimating equations to study sleep problems across the follow-up. General estimating equation models take the correlation of multiple measurements within one subject into account and yield an overall estimate of the association between early brain growth and repeatedly measured sleep problems. Moreover, they have an optimal 


\section{Articles | Kocesska e tal.}

Table 1. Children's brain development and sleep $(n=6,808)$

\begin{tabular}{l} 
Main determinants: brain growth \\
\hline Prenatal: mid pregnancy \\
Gestational age (weeks) \\
Estimated fetal weight $(\mathrm{g})$ \\
Head circumference $(\mathrm{mm})$ \\
Atrial width of lateral ventricles (mm) \\
Prenatal: late pregnancy \\
Gestational age (weeks) \\
Estimated fetal weight $(\mathrm{g})$ \\
Head circumference $(\mathrm{mm})$ \\
Atrial width of lateral ventricles (mm) \\
Postnatal \\
Age (weeks) \\
Weight (g) \\
Height (cm) \\
Head circumference $(\mathrm{mm})$ \\
Postnatal ultrasound $(n=813)$ \\
Age (weeks) \\
Head circumference $(\mathrm{mm})$ \\
Ventricular volume $(\mathrm{ml})$
\end{tabular}

$20.6 \pm 1.1$
$380.9 \pm 92.5$
$179.4 \pm 14.3$
$5.7 \pm 1.2$
$30.4 \pm 1.1$
$1,625.3 \pm 260.9$
$285.4 \pm 12.3$
$4.9 \pm 1.7$
$4.7 \pm 0.9$
$4,449.6 \pm 627.7$
$54.3 \pm 2.4$
$376.0 \pm 13.6$
$6.9 \pm 1.9$
$386.7 \pm 15.0$
$0.81(0.05-5.57)$

Outcomes: sleep patterns

Total sleep duration (h)

2 years of age

3 years of age

Dyssomnia symptoms, n(\%) "problematic sleepers"

1.5 years of age

3 years of age

6 years of age

\section{Child characteristics}

Sex, \% girls

Gestational age (weeks)

Birth weight $(\mathrm{g})$

Apgar score 5 min after birth

Ethnicity

Dutch (\%)

Other Western (\%)

Mediterranean (\%)

Caribbean (\%)

Non-western (\%)

Behavioral problems, score

\section{Maternal characteristics}

Age (years)

Parity (\% primipara)

Psychopathology score
49.7

$30.5 \pm 5.0$

57.5

$13.3 \pm 1.1$

$12.6 \pm 1.3$

1,213 (25.9)

$1,072(24.4)$

980 (16.9)

39.9 (25.3-43.6)

$3,441 \pm 551$

$10(2-10)$

58.6

8.6

13.3

9.7

9.8

$18(0.0-146.9)$

$.15(0.0-3.04)$
Table 1 Continued

\begin{tabular}{lc}
\hline Main determinants: brain growth & \\
\hline Smoking during pregnancy & 75.1 \\
\% No & 8.5 \\
\% Until pregnancy was known & 16.5 \\
\% Yes & \\
Educational level & 21.9 \\
\% Low & 30.6 \\
\% Medium & 47.5 \\
\% High & \\
\hline Numbers are percentages, means \pm SD or median (range) pooled from 10 imputed \\
data sets.
\end{tabular}

use of available measurements by allowing for incomplete outcome data. The baseline models were adjusted for (gestational) age at ultrasound measurement, sex, and head circumference (in the ventricular size models). Based on previous literature or a $>5 \%$ change in effect estimate of the predictor variable, the multivariable models were additionally adjusted for child's ethnicity, gestational age and Apgar score at birth, maternal age, parity, educational level, smoking, and psychiatric symptoms during pregnancy. To test whether the associations were influenced by body size, we additionally adjusted the models for estimated fetal weight (prenatal models), or birth weight and infant height and weight (postnatal models). In a final step, we additionally adjusted the models for child's behavioral problems (externalizing and internalizing problems scores in separate models), to test whether the observed associations were specific for sleep problems. In sensitivity analyses, we excluded children with $<3$ or $>3$ head circumference SD score in mid-pregnancy $(n=34)$, late pregnancy $(n=25)$, or postnatally $(n=21)$. We also tested if ventricular enlargement influenced our results by excluding fetuses with atrial width $>10 \mathrm{~mm}$ in midpregnancy $(n=2)$ or late pregnancy $(n=9)$, or neonates with lateral ventricular volume $3 \mathrm{SD}$ above the mean $(n=10)$. Missing values on covariates $(<10 \%)$ were imputed using multiple imputation to create 10 complete data sets. Statistical analyses were run in the 10 data sets and the results were pooled. For non-response analysis, several sociodemographic and maternal characteristics of children, who were lost to follow-up $(n=2,086)$, were compared $\left(\chi^{2}\right.$ test, $t$-test, or Mann-Whitney $U$-test) to those with available sleep data.

\section{RESULTS}

The baseline characteristics of the children included in this study are shown in Table 1 . Head circumference increased on average (SD) from $179(14.3) \mathrm{mm}$ to $286(12.3) \mathrm{mm}$ from mid- to late pregnancy, and further to $376(13.7) \mathrm{mm}$ at around 5 weeks after birth. The atrial width of the lateral ventricles decreased from 5.7 (1.2) $\mathrm{mm}$ in mid-pregnancy to $4.9(1.7) \mathrm{mm}$ in late pregnancy (Table 1). However, gestational age was not associated with the size of the ventricles within the third trimester $\left(\beta_{\text {gestational age, weeks }}=0.02\right.$, $P$ value $=0.954)$. The atrial width of the lateral ventricles in late pregnancy, however, was positively correlated with postnatal ventricular volume (Supplementary Table S2), which in turn increased further with postnatal age, i.e., older infants had a larger ventricular volume $\left(\beta_{\text {postnatal age, }}\right.$ ${ }_{\text {weeks }}=0.39, P$ value $\left.<0.001\right)$. Correspondingly, the correlation between different time points of ventricular size assessments were weak $(r=0.2)$ and decreased with time-lag (e.g., no 
correlation from mid-pregnancy to infancy). Average sleep duration (SD) decreased from $13.3 \mathrm{~h} \mathrm{(1.1)}$ at 2 years of age to $12.6 \mathrm{~h}(1.3)$ at 3 years of age.

\section{Brain Growth and Sleep Duration}

The associations of prenatal and early postnatal brain growth with sleep duration are shown in Table 2. Head circumference was not associated with sleep duration in any of the models. In contrast, larger lateral ventricles in the third trimester of pregnancy and also in early infancy were related to longer sleep duration. Both associations reached statistical significance with sleep duration at 3 years of age, but not at 2 years of age. Per 1-SD larger atrial width of the lateral ventricle in late pregnancy, sleep duration at age 3 years of age was $0.06 \mathrm{~h}$ (95\% confidence interval (CI): $0.02 ; 0.10$ ) longer. Consistently, per 1-SD larger ventricular volume in infancy, sleep duration was $0.11 \mathrm{~h}(95 \%$ CI: $0.02 ; 0.20)$ longer.
Importantly, these associations were not influenced by children with ventricular enlargement at any of the measurement rounds, and were not explained by fetal and infant body size or co-occurring behavioral problems (data not shown).

\section{Brain Growth and Repeated Measures of Sleep Problems}

Table 3 shows the association between prenatal and early postnatal brain growth and sleep problems across $1.5-6$ years of age. Fetuses with larger head circumference in midpregnancy were less likely to be "problematic sleepers" in early childhood, independent of child and maternal characteristics (OR: 0.94, 95\% CI: 0.89;0.99). Measures of brain growth during late pregnancy were also associated with later sleep problems. These associations, however, were largely explained by maternal characteristics such as educational level, smoking, and psychopathology symptoms during pregnancy. Individual time-point analyses shown in

Table 2. Associations between prenatal and early postnatal brain ultrasounds and sleep duration up to 3 years of age

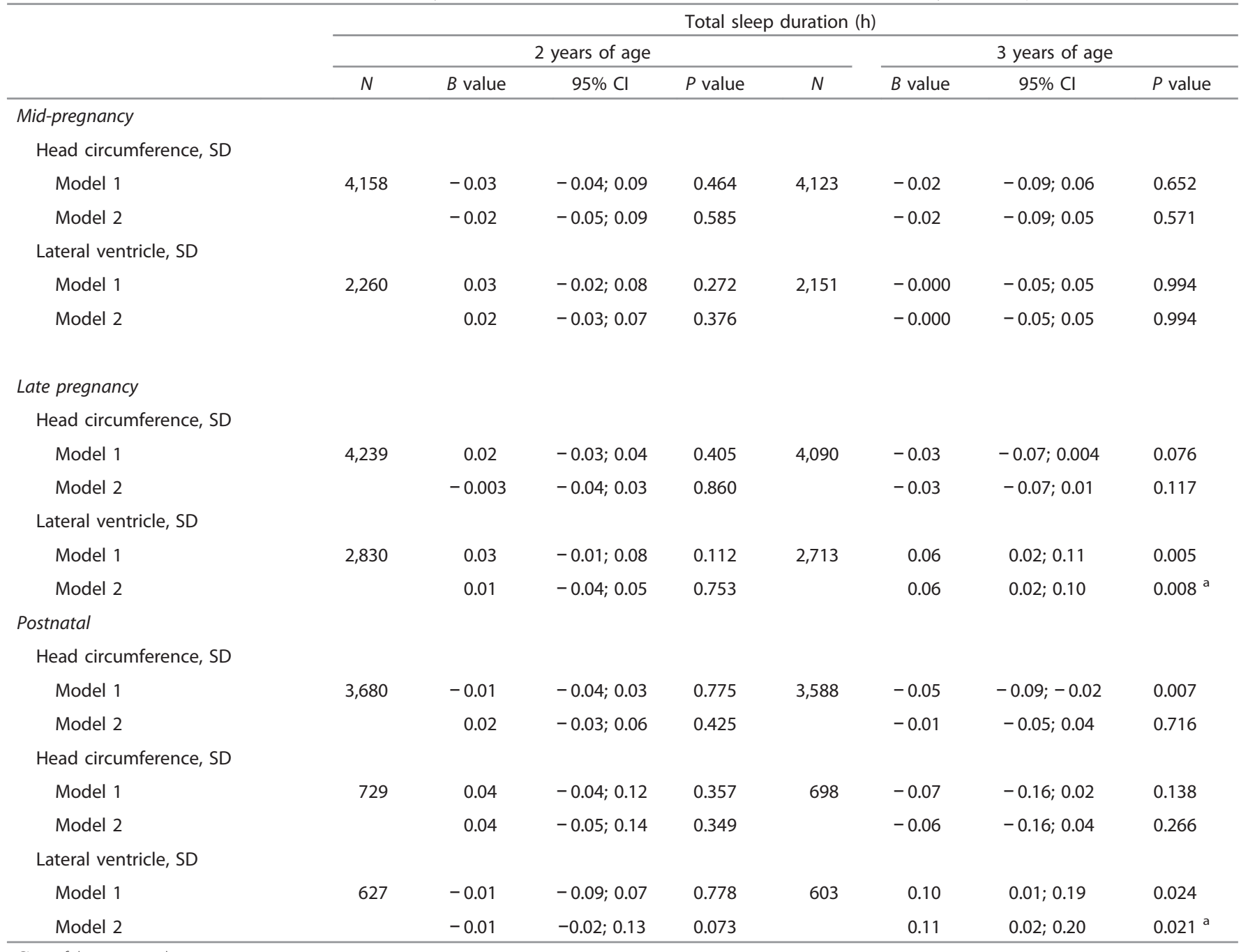

$\mathrm{Cl}$, confidence interval.

Model 1 is adjusted for gestational age at ultrasound assessment and sex, head circumference (ventricular volume models).

Model 2 is additionally adjusted for gestational age at ultrasound measurement, child's sex, ethnicity, gestational age and Apgar score at birth, maternal age, education, parity, psychopathology score, and smoking during pregnancy.

a Not explained by co-occurring behavioral problems or body size (estimated fetal weight in prenatal models, and birth weight or neonatal height and weight in postnatal models). 


\section{Articles | Kocerska et al.}

Table 3. Associations between brain measures and repeatedly measured sleep problems

\begin{tabular}{|c|c|c|c|c|c|}
\hline \multirow[t]{3}{*}{ Brain measures (z-scores) } & \multirow[t]{3}{*}{$N$} & \multicolumn{4}{|c|}{ 'Problematic sleepers' at 1.5, 3, and 6 years } \\
\hline & & \multicolumn{2}{|c|}{ Model 1} & \multicolumn{2}{|c|}{ Model 2} \\
\hline & & OR $(95 \% \mathrm{Cl})$ & $P$ value & OR $(95 \% \mathrm{Cl})$ & $P$ value \\
\hline \multicolumn{6}{|l|}{ Mid-pregnancy } \\
\hline Head circumference, SD & 5,825 & $0.91(0.88 ; 0.93)$ & $<0.001$ & $0.94(0.89 ; 0.99)$ & $0.013^{a}$ \\
\hline Lateral ventricle, SD & 2,843 & $0.98(0.95 ; 1.02)$ & 0.593 & $1.01(0.94 ; 1.08)$ & 0.782 \\
\hline \multicolumn{6}{|l|}{ Late pregnancy } \\
\hline Head circumference, SD & 5,951 & $0.90(0.88 ; 0.92)$ & $<0.001$ & $0.96(0.91 ; 1.02)$ & 0.169 \\
\hline Lateral ventricle, SD & 3,631 & $0.90(0.87 ; 0.93)$ & 0.002 & $0.95(0.89 ; 1.02)$ & 0.161 \\
\hline \multicolumn{6}{|l|}{ Postnatal } \\
\hline Head circumference, SD & 4,460 & $0.90(0.84 ; 0.96)$ & 0.002 & $0.95(0.89 ; 1.02)$ & 0.117 \\
\hline Head circumference, SD & 797 & $0.95(0.80 ; 1.11)$ & 0.516 & $0.97(0.83 ; 1.14)$ & 0.742 \\
\hline Lateral ventricles, SD & 689 & $0.99(0.82 ; 1.15)$ & 0.872 & $0.97(0.82 ; 1.15)$ & 0.758 \\
\hline \multicolumn{6}{|c|}{$\begin{array}{l}\mathrm{Cl} \text {, confidence interval; GEE, generalized estimating equation; OR, odds ratio. } \\
\text { Model 1: Adjusted for postconceptional age at ultrasound measure, gender, and head circumference (ventricles models). } \\
\text { Model 2: As Model 1, additionally adjusted for ethnicity, gestational age and Apgar score at birth, maternal educational level, parity, maternal smoking, and psychiatric symp- } \\
\text { toms during pregnancy. } \\
\text { ORs are derived from GEE. }\end{array}$} \\
\hline
\end{tabular}

Supplementary Tables S3 and S4 indicated that the observed longitudinal effects, were similar across the different ages of sleep assessment. However, larger head circumference in early infancy was associated with reduced risk of being a "problematic sleeper" at 3 years of age (odds ratio (OR): 0.89, 95\% CI: $0.80 ; 0.99)$ and 6 years of age(OR: $0.88,95 \%$ CI: $0.87 ; 0.99$ ), but not at 1.5 years of age (Supplementary Table S3 and S4). Despite violation of linearity assumption, similar results were obtained when sleep disturbance scores were analyzed continuously (Supplementary Table S4 and S5), indicating that cutoff points did not influence our results. Again, the observed associations between smaller head circumference and higher dyssomnia symptoms were not explained by behavioral problems, head circumference at the lower or upper extremes, or fetal size.

\section{Nonresponse Analysis}

Mothers of children included in the study were on average 3 years older, more likely to be Dutch $\left(32.6 \%\right.$ vs. $57.9 \%, \chi^{2} P$ value $<0.001)$, more highly educated $\left(19.5 \%\right.$ vs. $47.8 \%, \chi^{2} P$ value $<0.001)$, and had lower psychopathology scores during pregnancy (median score 0.43 vs $0.27, P<0.001$ ), compared to those lost to follow-up. In addition, children included in the study had larger head circumference than those lost to follow-up in mid- (mean difference $=0.18, P=0.001$ ) and late pregnancy (mean difference $=0.18, P<0.001$ ).

\section{DISCUSSION}

This study shows that prenatal and early postnatal ultrasonographic measures of brain development (i.e., head circumference and ventricular size) are related to sleep patterns across early childhood. Larger size of the ventricular system in late pregnancy and in early infancy were related to longer sleep duration at 3 years. In addition, larger head circumference in mid-pregnancy and early infancy was related to a reduced risk of being a "problematic sleeper" up to 6 years of age. The association between fetal brain measures in late pregnancy and later sleep problems was explained by maternal characteristics (e.g., mood disturbances during pregnancy). Albeit small, these effect estimates provide evidence for early neurodevelopmental origins of childhood sleep problems.

According to our knowledge this is the first study to explore prenatal brain growth as a determinant for later sleep patterns. Previous studies have reported that children born preterm have disturbed sleep patterns (9), which is compatible with the hypothesis that early neurodevelopment plays a role in the behavioral expression of sleep $(4,26)$. In addition, at least one longitudinal study has shown that adverse birth outcomes (e.g., low weight and/or length) are related to later sleep disturbances (6). Moreover, maternal psychopathology (8) and risky substance exposure during pregnancy (e.g., nicotine (6), alcohol (7), benzodiazepines (27)) have been related to short or disturbed sleep later in childhood.

Although the direct biological link between delayed neurodevelopment and disturbed sleep has not been established, sleep disturbances are highly prevalent among neurodevelopmental disorders, such as attention deficit hyperactivity disorder and autism $(28,29)$. Previous studies have also reported adverse prenatal brain development (e.g., smaller head circumference) among children with neurodevelopmental disorders $(30,31)$. However, whether differences in early brain development are also present in children with 


\section{Early head growth and sleep in childhood Articles}

disturbed sleep patterns is not known. Nevertheless, maturational delays in the sleep EEG have been observed in very-preterm infants ( $<32$ weeks of gestation) (32), in neonates with very-low birth weight $(32,33)$, and other perinatal complications (34). In addition, studies using fetal magnetography have shown that sleep-like behavioral states are tightly related to the neurodevelopmental stage of the fetus (35), and these states predict self-regulation in childhood and adolescence (4). This indicates that although the extrauterine environment influences sleep, maturation of sleep patterns is mainly a function of brain development.

In this study, we show that early markers of brain development are related to childhood sleep patterns, which tempts us to hypothesize that impaired neurodevelopment in prenatal or early postnatal life has a long-term effect on sleep regulation. Larger head circumference prenatally and in the first two postnatal months, indicating larger brain volumes, was related to a reduced risk of being a "problematic sleeper". Similar to other studies (36), the size of the ventricular system of the fetus showed a nonlinear relation with gestational age, resulting in a slight decrease of the average atrial width from the second to the third trimester of pregnancy followed by an increase thereafter. Only the size of the ventricular system in late pregnancy correlated positively with ventricular volume in early infancy. Postnatal ventricular volume may indicate advanced neural maturation as suggested by the positive correlation with age. Shortly before and after birth, larger ventricles within the normal range predicted longer sleep duration at 3 years of age, which in turn is developmentally beneficial. Importantly, ventricular enlargement due to medical complications has to be distinguished from larger ventricular volumes due to longer gestation and brain maturation. Previous studies by our group (10) as well as MRI studies (37) showed that in the general population a larger ventricular size in infancy indicates more advanced growth of the cerebral hemispheres. In addition, the size of the ventricular system is positively related to beneficial developmental outcomes (10) (e.g., larger ventricles before and shortly after birth were related to less temperamental difficulties (11)). As children with behavioral problems often have both adverse prenatal neurodevelopment (16) and sleep problems, (17) it is important that the results we report were not explained by co-occurring internalizing or externalizing problems. This means that early brain development is likely to have a specific link to disturbed sleep patterns, independent of comorbid mental conditions.

The mechanisms that play a role in the relation between early brain growth and childhood sleep patterns could be in line with the developmental origins of health and disease hypothesis (38). The brain tissue is a main substrate of the physiological and behavioral regulation of sleep (e.g., fetal rapid eye movement is considered to be an indicator and a promotor of brain development (39)), thus adversities in early neurodevelopment are likely to be reflected in disturbed postnatal sleep patterns. Along these lines, short sleep duration and dyssomnia symptoms might reflect developmental problems (22) that start prenatally and extend into childhood (5). Indeed, longitudinal research on sleep architecture in children and adolescents has shown that slow wave sleep is a reliable marker of cortical development and maturation $(5,33,40)$. Alternately, disturbances in the neuroendocrine properties of the fetal hypothalamic-pituitary-adrenal axis, which emerge towards the third trimester of pregnancy, could play a role in the relation between fetal brain growth and later sleep problems, including stress regulation, sleep, feeding, and emotion regulation (38).

Some methodological considerations need to be considered when interpreting our results. First, our results should not be generalized to clinical populations that have ventricular enlargement due to white matter damage or intraventricular hemorrhage. Second, the children included in our study had larger head circumference compared with those who were lost to follow-up, thus some selection bias could be present. Third, our study does not provide direct information about the growth trajectory of the nervous system. The postnatal measures were obtained only in an ethnically homogenous subsample, and the two-dimensional measures during pregnancy are not equivalent to the postnatal volumetric size of the ventricular system. Nevertheless, the atrial width of the lateral ventricle during pregnancy is predictive of the postnatal ventricular volume (10). Finally, maternal reports on children's sleep duration might have introduced some measurement error in our sleep estimates; however, we expect any outcome misclassification to be random (e.g., independent of brain development). In addition, early brain development might also influence other aspects of sleep (e.g., sleep efficiency), which would not be captured by maternal reports of sleep problems. Future studies should include objective measures of sleep to replicate and corroborate our findings. There are also some important advantages of our study, such as the longitudinal design with repeated measures of brain development and sleep patterns in a large sample of children from the general population. Importantly, because prenatal brain growth is a rapid process, our prenatal measures were standardized based on gestational age using study-specific growth curves (19). In addition, we were able to take key confounding factors into account, such as maternal smoking and psychopathology during pregnancy.

\section{Conclusion}

Understanding the development of sleep might be critical to understanding its functions. Previous research has mainly focused on the neurodevelopmental consequences of early childhood sleep disturbances, whereas this study shows that variations in fetal and neonatal brain development might underlie childhood sleep patterns. As sleep patterns mature along with the central nervous system, behavioral expressions of sleep might reflect neurodevelopment. Repeated measures using different imaging modalities within short time windows should be used to further elucidate the early neurobiological basis of sleep in childhood. 


\section{SUPPLEMENTARY MATERIAL}

Supplementary material is linked to the online version of the paper at http://www.nature.com/pr

\section{STATEMENT OF FINANCIAL SUPPORT}

The general design of Generation R Study is made possible by financial support from the Erasmus Medical Center, Rotterdam; the Erasmus University Rotterdam, Rotterdam; ZonMw; the Netherlands Organization for Scientific Research; and the Ministry of Health, Welfare, and Sport, and is conducted by the Erasmus Medical Center in close collaboration with the School of Law and Faculty of Social Sciences of the Erasmus University Rotterdam, the Municipal Health Service Rotterdam area, the Rotterdam Homecare Foundation, and the Stichting Trombosedienst and Artsenlaboratorium Rijnmond (STAR-MDC), Rotterdam. The first phase of the Generation R Study was made possible by financial support from the Erasmus Medical Centre and The Netherlands Organization for Health Research and Development (Zon MW Geestkracht Program 10.000.1003 and VIDI Grant 017.106.370 to H.T.). ERAWEB scholarship grant financed by the European Commission was granted to D.K. (grant agreement 20132548/001-001-EMA-2).

Disclosure: The authors declare no conflict of interest.

\section{REFERENCES}

1. Blumberg MD. Ontogeny of sleepKushida Ced Waltham, MA: Academic Press, 2013 pp 32-7.

2. Iglowstein I, Jenni OG, Molinari L, Largo RH. Sleep duration from infancy to adolescence: reference values and generational trends. Pediatrics 2003;111:302-7.

3. Stiles J, Jernigan TL. The basics of brain development. Neuropsychol Rev 2010;20:327-48.

4. Van den Bergh BR, Mulder EJ. Fetal sleep organization: a biological precursor of self-regulation in childhood and adolescence? Biol Psychol 2012;89:584-90.

5. Ringli M, Huber R. Developmental aspects of sleep slow waves: linking sleep, brain maturation and behavior. Prog Brain Res 2011;193:63-82.

6. Pesonen AK, Raikkonen K, Matthews K, et al. Prenatal origins of poor sleep in children. Sleep 2009;32:1086-92.

7. Troese M, Fukumizu M, Sallinen BJ, et al. Sleep fragmentation and evidence for sleep debt in alcohol-exposed infants. Early Hum Dev 2008;84:577-85.

8. O'Connor TG, Caprariello P, Blackmore ER, et al. Prenatal mood disturbance predicts sleep problems in infancy and toddlerhood. Early Hum Dev 2007;83:451-8.

9. Huang YS, Paiva T, Hsu JF, Kuo MC, Guilleminault C. Sleep and breathing in premature infants at 6 months post-natal age. BMC Pediatr 2014;14:303.

10. Roza SJ, Govaert PP, Vrooman HA, et al. Foetal growth determines cerebral ventricular volume in infants. The Generation R Study. Neuroimage 2008;39:1491-8.

11. Roza SJ, Govaert PP, Lequin MH, et al. Cerebral ventricular volume and temperamental difficulties in infancy. The Generation R Study. J Psychiatry Neurosci 2008;33:431-9.

12. Gilmore JH, Kang C, Evans DD, et al. Prenatal and neonatal brain structure and white matter maturation in children at high risk for schizophrenia. Am J Psychiatry 2010;167:1083-91.

13. Gilmore JH, Smith LC, Wolfe HM, et al. Prenatal mild ventriculomegaly predicts abnormal development of the neonatal brain. Biol Psychiatry 2008;64:1069-76.

14. Kyriakopoulou V, Vatansever D, Davidson A, et al. Normative biometry of the fetal brain using magnetic resonance imaging. Brain Struct Funct 2016;222:2295-307.

15. Heinonen K, Raikkonen K, Pesonen AK, et al. Prenatal and postnatal growth and cognitive abilities at 56 months of age: a longitudinal study of infants born at term. Pediatrics 2008;121:e1325-33.
16. Schlotz W, Godfrey KM, Phillips DI. Prenatal origins of temperament: fetal growth, brain structure, and inhibitory control in adolescence. PLoS ONE 2014;9:e96715.

17. Gregory AM, Sadeh A. Annual Research Review: sleep problems in childhood psychiatric disorders-a review of the latest science. J Child Psychol Psychiatry 2016;57:296-317.

18. Kooijman MN, Kruithof CJ, van Duijn CM, et al. The Generation R Study: design and cohort update 2017. Eur J Epidemiol 2016;31:1243-64.

19. Verburg BO, Steegers EA, De Ridder M, et al. New charts for ultrasound dating of pregnancy and assessment of fetal growth: longitudinal data from a population-based cohort study. Ultrasound Obstet Gynecol 2008;31:388-96.

20. Dutch Growth Research Foundation Growth Analyzer 3.0. Rotterdam, The Netherlands: Dutch Growth Research Foundation, 2007.

21. Achenbach TM, Rescorla LA Manual for the ASEBA Preschool Forms and Profiles. In: Research Center for Children Youth, and Families. Burlington, VT: University of Vermont, 2000 pp 55-68.

22. Kocevska D, Muetzel R, Luik AI, et al. The developmental course of sleep disturbances across childhood relates to brain morphology at age seven. The Generation R Study. Sleep 2016;40(1).

23. Hadlock FP, Harrist RB, Sharman RS, Deter RL, Park SK. Estimation of fetal weight with the use of head, body, and femur measurements-a prospective-study. Am J Obstet Gynecol 1985;151:333-7.

24. Netherlands Statistics 2006 www.cbs.nl.

25. De Beurs E. Brief Symptom Inventory-Manual 2004.

26. Kinsella MT, Monk C. Impact of maternal stress, depression and anxiety on fetal neurobehavioral development. Clin Obstet Gynecol 2009;52: 425-40.

27. Bourke $\mathrm{CH}$, Stowe $\mathrm{ZN}$, Owens MJ. Prenatal antidepressant exposure: clinical and preclinical findings. Pharmacol Rev 2014;66:435-65.

28. Scott N, Blair PS, Emond AM, et al. Sleep patterns in children with ADHD: a population-based cohort study from birth to 11 years. J Sleep Res 2013;22:121-8.

29. Humphreys JS, Gringras P, Blair PS, et al. Sleep patterns in children with autistic spectrum disorders: a prospective cohort study. Arch Dis Child 2014;99:114-8.

30. Kaushik G, Zarbalis KS. Prenatal neurogenesis in autism spectrum disorders. Front Chem 2016;4:12.

31. Heinonen K, Räikkönen K, Pesonen A-K, et al. Trajectories of growth and symptoms of attention-deficit/hyperactivity disorder in children: a longitudinal study. BMC Pediatr 2011;11:84.

32. Nunes ML, Khan RL, Gomes Filho I, Booij L, da Costa JC. Maturational changes of neonatal electroencephalogram: a comparison between intra uterine and extra uterine development. Clin Neurophysiol 2014;125: 1121-8.

33. Wehrle FM, Latal B, O'Gorman RL, Hagmann CF, Huber R. Sleep EEG maps the functional neuroanatomy of executive processes in adolescents born very preterm. Cortex 2017;86:11-21.

34. Aldrete-Cortez V, Schnaas L, Poblano A, et al. Effect of late-onset fetal growth restriction on organization of behavioral state in infants. Pediatr Int. 2015;57:902-8.

35. Kiefer-Schmidt I, Raufer J, Brandle J, et al. Is there a relationship between fetal brain function and the fetal behavioral state? A fetal MEG-study. J Perinatol Med. 2013;41:605-12.

36. Salomon LJ, Bernard JP, Ville Y. Reference ranges for fetal ventricular width: a non-normal approach. Ultrasound Obstet Gynecol 2007;30:61-6.

37. Knickmeyer RC, Gouttard S, Kang C, et al. A structural MRI study of human brain development from birth to 2 years. J Neurosci 2008;28: 12176-82.

38. Lewis AJ, Galbally M, Gannon T, Symeonides C. Early life programming as a target for prevention of child and adolescent mental disorders. BMC Med 2014;12:33.

39. Mirmiran M. The function of fetal/neonatal rapid eye movement sleep. Behav Brain Res 1995;69:13-22.

40. Buchmann A, Ringli M, Kurth S, et al. EEG sleep slow-wave activity as a mirror of cortical maturation. Cereb Cortex 2011;21:607-15. 